\title{
One Health in Action: Lyme Disease
}

\author{
Veronica A. Fialkowski, Erik Foster, Kim Signs and Mary Grace Stobierski \\ Emerging and Zoonotic Infectious Diseases Section, Michigan Department of Health and Human Services, Lansing, MI, USA
}

\section{Introduction}

Lyme disease is an emerging disease in Michigan and is the most commonly reported vector-borne illness. The bacterium causing Lyme disease, Borrelia burgdorferi, is transmitted to humans or dogs through the bite of an infected tick. In the spring of 2015, a veterinarian from an island on Lake Michigan began to see locallyacquired Lyme disease in pets. In previous years the vector of Lyme disease, Ixodes scapularis, had not been found on the island. The Michigan Department of Health and Human Services (MDHHS) was invited to the island to conduct an environmental investigation and provide health education to local residents.

\section{Methods}

To determine the tick population on the island, tick drags were conducted, which is a method for collecting ticks. The method involves dragging a 1-square-meter strip of white cloth mounted on a dowel tied to rope through terrain that may harbor ticks. Tick drags were done in multiple locations on the island.

\section{Results}

Multiple life stages of Ixodes scapularis were found on the island. In total, 24 I. scapularis ticks were collected on the day of the island visit and two were positive ( 8.3 percent) for B. burgdorferi. Health education was presented at a town meeting and included information about Lyme disease, the vector for the disease, and methods on how to prevent tick-borne disease in humans and pets. "Ticks and your health" brochures and tick identification cards were provided to the residents.

\section{Conclusions}

The intersection of people, animals, and the environment is well represented in this response to public concern of Lyme disease. Animal health surveillance for Lyme disease prompted environmental surveillance for ticks and led to proactive health education. By collaborating effectively with individuals from many fields, the One Health approach allowed for a comprehensive response of the emergence of Lyme disease on the island.

\section{Keywords}

One Health; Lyme disease; Ticks

\section{Acknowledgments}

Dr. Jeffrey Powers, DVM

Dr. Meghan Weinberg, PhD, MPH; Michigan Department of Health and Human Services

Dr. Jennifer Sidge, DVM, Michigan State University

Dr. Jean Tsao, PhD, Michigan State University

Dr. Graham Hickling, PhD, University of Tennessee

This study/report was supported in part by an appointment to the Applied Epidemiology Fellowship Program administered by the Council of State and Territorial Epidemiologists (CSTE) and funded by the Centers for Disease Control and Prevention (CDC) Cooperative Agreement Number 1U38OT000143-02

*Veronica A. Fialkowski

E-mail: FialkowskiV@michigan.gov 